\title{
Planning ahead: Improving escalation plans before the weekend
}

\author{
Angeliki Zarkali, Duncan Black, Elizabeth Smee, Anshul Deshraj, Nicholas Smallwood \\ Royal Surrey County Hospital
}

\begin{abstract}
Handover is the system by which responsibility for patient care is transferred between healthcare professionals. A significant aspect of handover is the existence of an escalation plan for each patient in case of deterioration over the weekend. According to the Royal College of Physicians, all patients should have a clear escalation plan documented in the notes before a weekend, since parent medical teams (Consultant team in charge of care) are best placed to make these decisions. If left to on-call teams, at a time of deterioration over a weekend, they might not have all available information, the patient might be unable to be involved in the decisions, and the family might not be consulted.
\end{abstract}

With this is mind, we decided to analyse the existing handover process in a medium sized district general hospital, with the aim of improving the process and the documentation of escalation plans. The results from our retrospective analysis of the system in place revealed a significant lack of documentation of escalation plans in the medical notes.

Three sample wards were selected to analyse the current handover system and test proposed measures before hospital-wide implementation. After trialling of a physical handover meeting in addition to the existing intranet system and a proforma for the Friday ward round, the documentation of escalation plans in the patients' notes improved from $9.1 \%$ to $41.1 \%$. Based on these results, as well as formal feedback from junior doctors and informal feedback from other staff, the physical handover meeting and Friday ward round proforma will be implemented throughout the Trust.

Our interventions led to an improvement in the documentation of escalation plans in our hospital, thus saving precious time in the event of a patient's deterioration. This also ensures that families and patients are involved in the decision making process and kept informed, and reduces the burden for the weekend on-call teams.

\section{Problem}

Handover is a significant process in medicine that ensures patient safety, especially before the weekend. One significant aspect of handover is the documentation of escalation plans in case of deterioration over the weekend. We found that documentation of escalation planning in our medium sized (527 bed) district general hospital $(\mathrm{DGH})$ was poor before the weekend and not handed over appropriately to the on-call team. This led to important decisions being made by doctors who may not have all the appropriate information, and potentially difficult escalation and resuscitation decisions not being made by teams who know the patients best.

In a survey of junior doctors that we conducted in our hospital, we found that junior doctors are only aware of the escalation plans for "a few" of their patients; thus they document these plans only occasionally in the patients' notes on the ward round on Friday. In addition, junior doctors reported that when they are asked to see a patient over the weekend, only rarely is an escalation plan documented in the patient's notes. It was therefore apparent that an improvement in the documentation of escalation decisions before the weekend would benefit on-call teams and potentially improve patient care.

\section{Background}

Handover is the system by which responsibility for patient care is transferred between healthcare professionals. The number of handovers occurring during a typical working week has increased as a result of the increase in shift working due to the European Working Time Directive. This means that handovers have become a major preventable cause of patient harm as identified by the National Patient Safety Agency, the Royal College of Physicians (RCP), the British Medical Association, and the General Medical Council (GMC) $(1,2,3,4)$.

A study by Bhabra et al (5) found that over a typical weekend there will be up to five shift handovers; only $2.5 \%$ of information from the first handover is retained to the final handover if there is no written record. If notes are taken $85.5 \%$ of information is retained. This rises to $99 \%$ if a standardised proforma is used .

Poor handover is a major preventable cause of patient harm and is due to human factors of poor communication and systemic error (2). The GMC and the Foundation Programme consider handover so important that it has included it as a compulsory part of the curriculum (6). This means that improvements in handover are essential for increasing patient safety and are required for good medical practice.

One of the most significant aspects of handover is the escalation planning in the case of deterioration over the weekend, as this 
decision needs to take several aspects of the patient's case into account, is time-consuming, and should ideally involve the patient and his or her family. As per advice from the RCP, all patients should have a clear escalation plan documented in the medical notes in the case of deterioration before a weekend, since parent medical teams are best placed to make these decisions. If it is left to on-call teams at a time of deterioration over a weekend, they might not have all available information, the patient might be unable to be involved in the decisions, and the family might not be consulted.

\section{Baseline Measurement}

We performed a retrospective analysis of all patients' notes in three sample general medical wards of our DGH as well as the handover sheet for these wards for the weekend of 27-28 April 2013. All patients who were new admissions and were seen 'post-take' (reviewed by a consultant) on Friday morning were excluded, as it was felt that the day team would be unfamiliar with these patients.

These three wards were chosen to represent the inpatient population of the hospital, with a combination of a respiratory ward (comprising usually unstable or acutely unwell patients, thus a high priority when it comes to making escalation decisions and weekend plans), a geriatric ward (involving a mix of medically stable patients with social needs and acutely unwell patients), and a mixed ward with both high and low priority patients.

A total of 77 patients were screened on these three wards on Monday 29 April 2013. Eleven of these patients were new admissions and were 'post-taked' (reviewed by a consultant) on Friday, and thus were excluded from further analysis.

The mean age of the patients was 79.2 years. Regarding escalation plans for these patients, a significant percentage $(40.9 \%)$ of them were of 'Do not resuscitate' status; however, only $9.1 \%$ had clear escalation plans documented during the Friday ward round, for example, not for ventilation, for full escalation or ward management only, etc.

Our Critical Care Outreach Team (CCOT; the specialist nurse-led team which responds to emergency calls in the hospital and provides advice in the case of a patient's deterioration), reviewed two patients over the weekend, none of whom was transferred to the intensive therapy unit (ITU). There was one death over the weekend and the on-call registrar made the decision not to escalate. For this patient there were no documented escalation plans in the Friday ward round.

Only 38 of the 66 patients (57.6\%) had a weekend plan documented in the notes during the Friday ward round. Of these 38 patients, 26 had it documented in the notes that a job needed to be done over the weekend, but only 18 patients had that documented in the handover sheet. There is a significant inconsistency in the jobs that the medical teams hand over to the on-call team, with $30.8 \%$ of the jobs documented in the Friday ward round not being handed over to the weekend team. Only nine patients had a plan documented in the notes to be followed in case the patient deteriorated.

\section{Design}

When investigating the reasons for the lack of documentation of escalation plans, it became clear that junior doctors who are documenting the ward round and handing over to the weekend team are often not aware of these escalation plans themselves, and are reluctant or forget to ask their seniors regarding these plans. In order to overcome this barrier, we designed a proforma for the Friday ward round where escalation plans and jobs for the on-call team would be clearly recorded. In addition a physical handover meeting was proposed and trialled as an adjunct to the current formintranet based system.

Before trialling any intervention, we performed a survey of the junior doctors in medical teams where the current handover system and potential alternatives were discussed. The survey showed that the majority of junior doctors ( $69.7 \%$ of total responders) thought a physical handover would be helpful. Those who objected said it would be difficult to get the necessary people to meet and that it would take a lot of time.

In addition, $90.9 \%$ of responders thought that a short proforma would improve the weekend handover, but they commented on the need for this proforma to be short and kept in the patients' notes due to the increased paperwork that already needs to be completed on Fridays.

Taking this feedback into account, we designed the Friday proforma as a single page that would replace the normal medical notepaper every Friday and would not require any other additional documentation.

\section{Strategy}

PDSA cycles 1-2. Production of the Friday ward round proforma. The proforma was discussed in a group of junior doctors and changes were made in order to ensure that the proforma is short, easy to use, and that no extra paperwork would need to be completed.

PDSA cycle 3. All junior doctors, consultants, ward managers and nursing staff (both senior and junior levels of nursing) in the three trial wards were informed and made familiar with the new Friday ward round proforma.

PDSA cycle 4. A physical handover meeting was trialled on Friday afternoon. The meeting was attended by the consultant, registrar, Foundation Year 1 (FY1) and Senior House Officer (SHO) who would be on call over the weekend as well as the outreach team, ITU staff, and the bed managers. At the meeting a representative of each medical team presented details of the sick patients on their ward and their escalation plans and potential weekend discharges.

\section{Results}


During the second cycle of the audit, a total of 72 patients were screened on Monday 22 July 2013. Sixteen of these patients were new admissions, post-taked on Friday, and thus were excluded from further analysis.

Of the remaining 56 patients, the proforma was used in $33.9 \%$ (19 patients) and the mean age was 81.3 years. $48.2 \%$ of the patients were 'not for resuscitation', slightly higher than the $40.9 \%$ in the first cycle; one patient was documented as 'not for resuscitation' but the DNAR form was to be completed at a later stage because the family had not been contacted.

The percentage of patients with documented escalation plans was also significantly higher at $41.1 \%$ (27 patients) compared to only $9.1 \%$ in cycle 1 . Of the 23 patients who had a documented escalation plan in place, 10 were for 'full escalation', nine for ward based care only, three were recorded as awaiting decision or discussion with family, and one had non-invasive ventilation (NIV) as the ceiling of care.

\section{Lessons and Limitations}

A strength of this quality improvement project, and one of the main factors that made our intervention successful, was that the proposed intervention was discussed and supported by the majority of the junior doctors in the hospital $(69.7 \%$ of responders would like a physical handover and $90.9 \%$ a Friday proforma). Performing a survey of the junior doctors to get their feedback on possible solutions was a valuable step in the process and ensured that our interventions would be welcomed and used by the medical teams.

Another significant strength of the project was the involvement of other healthcare professionals and doctors from other specialties in the process, especially the outreach team, bed managers, and the ITU team. The physical handover meeting was attended by all these disciplines in addition to the medical team. This was of great value for the on-call team, who found it easier to refer a patient to CCOT or ITU in case of deterioration as in most cases they were already aware of the patient and escalation plan. It was also valuable for the ITU staff as they were aware of all the acutely unwell patients in the hospital.

There are certain limitations to our project. First, although the feedback was positive and the re-audit showed change, the Friday ward round proforma was used in only $33.9 \%$ of the patients we reviewed. This lack of engagement was particularly evident in one of the three trial wards where the form was only used in a few patients. After discussions with the junior doctors of that ward, they reported problems with the supply of the form, as well as time constraints, increased workload due to sickness, and hesitation in probing their seniors for escalation plans. We have tried to resolve some of these issues by educating both junior and senior medical staff again, and ensuring the form is easily accessible. although they are thought to be representative, the results might be different when the change is implemented throughout the hospital. This is currently being undertaken in collaboration with senior medical and nursing staff to ensure a consistent approach.

\section{Conclusion}

The institution of a Friday ward round proforma has led to a significant improvement in the documentation of escalation plans before the weekend in our hospital. Having an escalation plan in place in case of deterioration is believed to lead to more appropriate decisions, as the clinicians who make these decisions during normal working hours are more likely to be senior, to know the patient better, and to consult the family, compared to clinicians who make these decisions while on-call. It will also lead to increased patient satisfaction as the patient and the family are more involved and better informed about these plans, and will relieve some pressure on the already busy on-call team. It will also likely ensure that the most appropriate decision is made in any given situation, ensuring patients and families are treated with care and dignity when stressful changes in conditions occur out of normal working hours.

\section{References}

1. National Patient Safety Agency. Seven steps to patient safety. London: NPSA, 2004.

2. Royal College of Physicians. Acute Care Toolkit 1 : Handover. London: RCP, May 2011.

3. General Medical Council. Tomorrow's doctors. Outcomes and standards for undergraduate medical education. London: GMC, 2009

4. British Medical Association. Safe handover: safe patients. Guidance on clinical handover for clinicians and managers. London: BMA, 2004.

5. Bhabra G, Mackeith S, Monteiro P, Pothier D. An experimental comparison of handover methods. Ann R Coll Surg Engl 2007;89: 298-300.

6. The UK Foundation Programme. Curriculum. Cardiff: UKFPO, 2012

\section{Declaration of interests}

None declared

\section{Acknowledgements}

To all staff in the hospital who provided us with their feedback and support
Another limitation of our report is that data were collected during only one weekend on two separate occasions. Further measurements are necessary to confirm the impact of these measures. Finally, the proforma was trialled in three wards, and 